\title{
DETERMINACIÓN DE PLANTAS HOSPEDANTES ALTERNAS DE Steneotarsonemus spinki EN ZONAS ARROCERAS DE COSTA RICA
}

\author{
Jean Alexander Gamboa Tabares ${ }^{1}$, Ruth León González², Víctor Manuel Cartín Leiva ${ }^{3}$, \\ Francisco Álvarez Bonilla ${ }^{4}$, Israel Garita Cruz ${ }^{5}$
}

\begin{abstract}
RESUMEN
Steneotarsonemus spinki constituye una de las plagas de reciente aparición y de las más notables para el arroz en Costa Rica. El objetivo de este trabajo fue determinar en campo las arvenses, asociadas a campos de arroz en Costa Rica, que sirven de hábitat al ácaro $S$. spinki. Entre agosto y septiembre, 2007, se tomaron muestras de plantas asociadas a 16 campos de arroz Oryza sativa, en cuatro zonas arroceras: Brunca, Pacífico Central, Huetar Atlántico y Chorotega; afectadas por altas densidades poblacionales del ácaro S. spinki. Con la ayuda de estereoscopio se determinó la presencia de $S$. spinki en las especies arvenses Echinochloa colona, Eleusine indica, Oryza latifolia y Rottboellia cochinchinensis; y densidades poblacionales de 0,$4 ; 0,1 ; 13,2$ y 0,2 ácaros/ planta, respectivamente.
\end{abstract}

Palabras clave: Oryza sativa, Steneotarsonemus spinki, plantas hospedantes.

\section{NTRODUCCIÓN}

Los tarsonémidos constituyen uno de los grupos de ácaros de mayor importancia para el hombre en la agricultura por constituir plagas de muchos cultivos. Entre los géneros de más interés se encuentra Steneotarsonemus, de amplia distribución mundial, y el segundo en número de especies (60) dentro de la familia.
Se ha informado de varias especies de ácaros pertenecientes a la familia Tarsonemidae que se encuentran en arroz: Steneotarsonemus spinki Smiley, Steneotarsonemus furcatus De León y Steneotarsonemus spirifex Marchal (Reyes 2005). El ácaro fitófago S. spinki se encontró en Costa Rica en agosto de 2004 (Barquero 2004) como parte del complejo de organismos causantes del vaneado de la panícula y la pudrición de la vaina de arroz.

\footnotetext{
${ }^{1}$ Tesis sometida a consideración del Tribunal Examinador del Sistema de Estudios de Posgrado de la Universidad Nacional para optar al grado de Magíster Scientiae en Agricultura Alternativa con mención en Agricultura Ecológica. Ingeniero Agroecólogo. Docente-investigador Universidad de la Amazonia. Correo electrónico: gamboatabares @ gmail.com.

2 Instituto Nacional de Investigación e Innovación y Transferencia en Tecnología Agropecuaria (INTA), Correo electrónico: rleon@inta.go.cr Dpto. Investigación e Innovación Tel.:2231- 5055.

${ }^{3}$ Escuela de Ciencias Agrarias, Universidad Nacional. Te.: 2277-3298. Correo Electrónico: vcartin @ una.ac.cr.

${ }^{4}$ Equipo de Extensión Agrícola. Dirección Regional Chorotega. Ministerio de Agricultura y Ganadería. Liberia, Costa Rica.

${ }^{5}$ Gerente de Investigación Duwest Inc. Centroamérica y Caribe. Correo electrónico: Israel.Garita@ duwest.com. Apartado postal: 219-3009 Santa Bárbara de Heredia, Costa Rica.
} 
El ácaro S. spinki se establece en las plantas y con ayuda de sus quelíceros succiona directamente el contenido de células epidérmicas de las hojas, lo que disminuye la capacidad fotosintética y produce alteraciones en el crecimiento de las plantas. En las hojas afectadas se incrementa agudamente la transpiración, se rompe el balance hídrico y se interrumpe el proceso de fotosíntesis. Además, provoca daños indirectos al ser diseminador de hongos y bacterias; así como por la inyección de tóxicos en el proceso de alimentación. Se ha observado que en los daños mecánicos producidos por este fitófago aparecen síntomas muy severos de organismos oportunistas que agudizan el cuadro de daño y pérdidas de calidad y cantidad de la producción (Almaguel 2002)

En los países afectados por este ácaro, se recomienda la implementación de métodos de manejo integrado basados fundamentalmente en la eliminación de los restos de cosechas y de arvenses asociadas al cultivo, uso racional de los fertilizantes nitrogenados, conservación y aumento de los enemigos naturales, siembra de los campos colindantes con un período no menor de tres semanas de diferencias y empleo de variedades resistentes, y como última alternativa se plantea la utilización de productos químicos. Este método de manejo integrado de plagas (MIP) es definido por Hilje (1995) como una noción o estrategia, de carácter preventivo y perdurable, que combina varias tácticas compatibles para reducir las poblaciones de organismos a niveles que no causen pérdidas económicamente importantes, con efectos negativos mínimos sobre el ambiente o la salud humana.

En lo referente a la posible relación $S$. spinki - plantas hospedantes, es necesario analizar cuán adecuada es cada planta hospedante para la supervivencia, desarrollo y reproducción del ácaro, lo que en gran parte define el potencial para aumentar sus poblaciones. Posteriormente, es conveniente estudiar la biología de $S$. spinki sobre las especies hospedantes y así poder determinar el papel que juega cada especie en la problemática provocada por el ácaro en los agroecosistemas arroceros costarricenses. $Y$ un tercer componente relacionado con este problema, es el efecto que cada planta hospedante pueda tener sobre la interacción del ácaro fitófago y sus enemigos naturales (interacción de tres niveles tróficos)

Las plantas hospedantes de $S$. spinki registradas en la literatura para diferentes países, incluyen 11 especies de plantas pertenecientes a tres familias botánicas: Eleusine indica (L.) Gaertn, Lingnania chungii (McClure) McClure, Schizostachyum funghomii McClure, Imperata cylindrica (L.) Raeusch, Leersia hexandra Sw., Paspalum sp., Oryza latifolia Desv., Echinochloa colona (L.) Link, y Panicum purpuracens Raddi, de la familia Poaceae; Amaranthus spinosus L., de la familia Amaranthaceae; y Schoenoplectus articulatus (Linn.) Palla, de la familia Cyperaceae. Muchos de estos registros no indican datos referenciales ni procedencia de la información sobre el material estudiado, por lo que la información a ser corroborada resulta insuficiente.

El estudio de plantas hospedantes contribuirá a definir la importancia de éstas en el manejo integrado de ácaros plagas y en la toma de decisiones para el manejo y combate de S. spinki. Por estas razones, la presente investigación ha propuesto determinar las plantas asociadas a campos de arroz en Costa Rica, que sirven de hábitat al ácaro S. spinki.

\section{MATERIALES Y MÉTODOS}

El estudio en campo de plantas hospedantes de $S$. spinki se realizó durante los meses de agosto y septiembre de 2007. Se evaluaron 16 campos de arroz, distribuidos en cuatro regiones arroceras de Costa Rica: Brunca, Pacífico Central, Huetar Atlántico y Chorotega. En la Figura 1 y el Cuadro 1, se indican algunas características ambientales de los predios muestreados en cada región arrocera. 


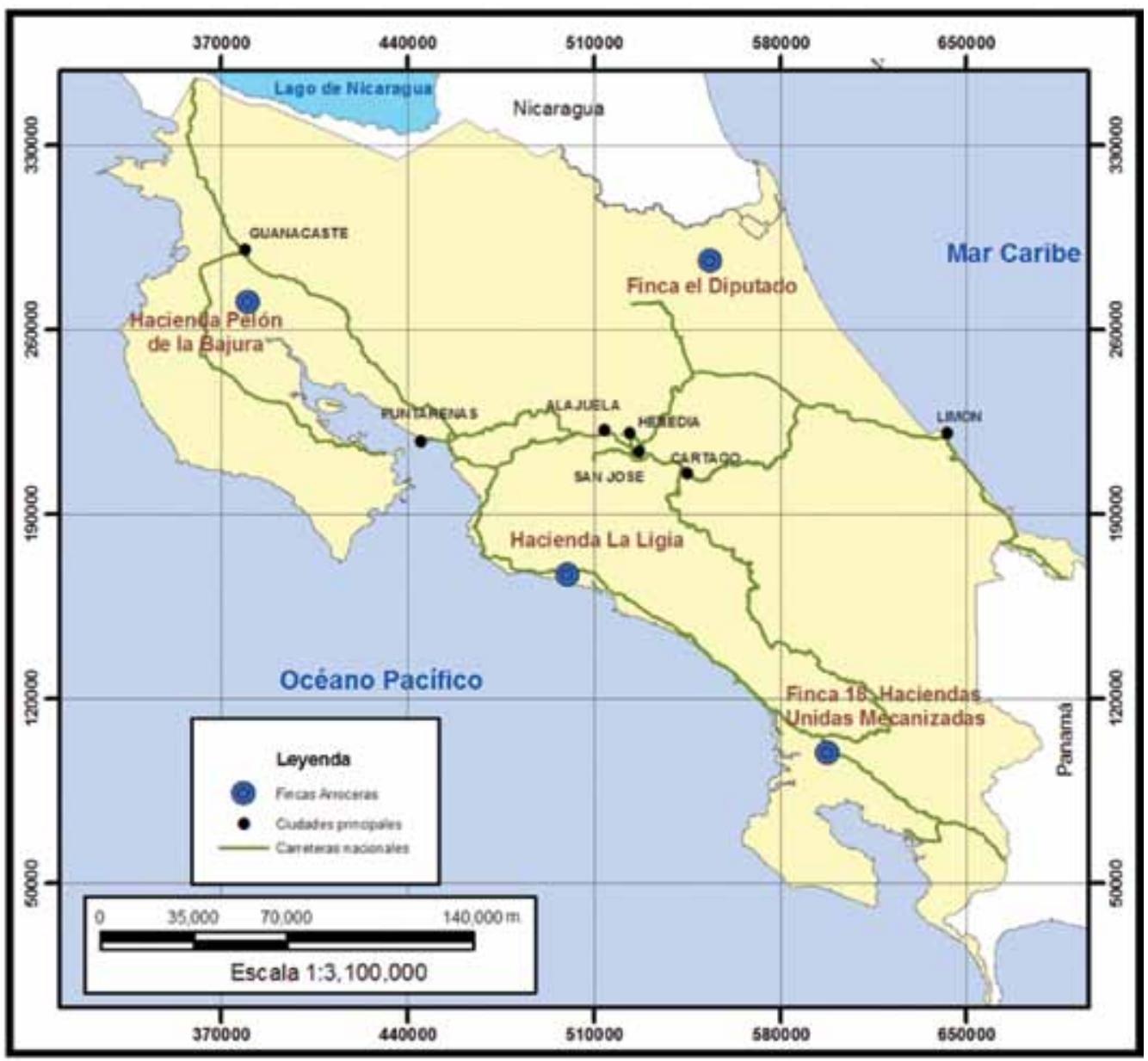

Figura 1. Ubicación de las fincas arroceras muestreadas en Costa Rica. Agosto-Septiembre, 2007.

Cuadro 1. Características ambientales de los lugares de muestreo. En las cuatro fincas arroceras muestreadas en Costa Rica. Agosto-Setiembre, 2007.

\begin{tabular}{|c|c|c|c|c|c|c|c|}
\hline Región & Provincia & Cantón & Fincas & $\begin{array}{l}\text { Altitud } \\
\text { (msnm) }\end{array}$ & $\begin{array}{l}\text { Precipitación } \\
\text { promedio } \\
\text { anual }(\mathrm{mm})\end{array}$ & $\begin{array}{l}\text { Temperatura } \\
\text { promedio } \\
\text { anual }\left({ }^{\circ} \mathrm{C}\right)\end{array}$ & $\begin{array}{c}\text { Zona de vida según } \\
\text { clasificación de } \\
\text { Holdridge * }\end{array}$ \\
\hline Brunca & Puntarenas & Osa & $\begin{array}{c}\text { Finca } 18 \\
\text { Haciendas } \\
\text { Unidas } \\
\text { Mecanizadas }\end{array}$ & $0-100$ & 4.000 & 25 & bmh-P6 \\
\hline $\begin{array}{l}\text { Pacífico } \\
\text { Central }\end{array}$ & Puntarenas & Parrita & La Ligia & $0-100$ & 3.000 & 25 & bh-T2 \\
\hline $\begin{array}{c}\text { Huetar } \\
\text { Atlántico }\end{array}$ & Heredia & Sarapiquí & El Diputado & $0-100$ & 4.500 & 25 & bmh-T \\
\hline Chorotega & Guanacaste & $\begin{array}{l}\text { Liberia- } \\
\text { Bagaces }\end{array}$ & $\begin{array}{l}\text { Hacienda } \\
\text { Pelón de } \\
\text { la Bajura }\end{array}$ & $0-100$ & 2.000 & 27 & bh-P6 \\
\hline
\end{tabular}

Fuente: Holdridge (1993).

* bmh-P6: bosque muy húmedo premontano transición a basal; bh-T2: bosque húmedo tropical transición a perhúmedo, bmh-T: bosque muy húmedo tropical, bh-P6: bosque húmedo premontano transición a basal. 


\section{Muestreo}

La selección de los campos de arroz muestreados se basó en dos condiciones: arroz donde hubo ácaros S. spinki en la cosecha anterior y campos donde estaba el ácaro en plantas de arroz al momento de muestreo. El Cuadro 2 resume algunas características de los campos muestreados.

Cuadro 2. Características de los campos muestreados en distintas regiones arroceras de Costa Rica. AgostoSetiembre, 2007.

\begin{tabular}{|c|c|c|c|c|}
\hline No. & Predio & $\begin{array}{c}\text { Nombre de } \\
\text { campo muestreado }\end{array}$ & Área (Hectáreas) & $\begin{array}{l}\text { Condición } \\
\text { de Campo }\end{array}$ \\
\hline 1 & Finca 18 H.U.M & 24 & 151,54 & Floración \\
\hline 2 & Finca 18 H.U.M & 22 & 44,07 & Maduración \\
\hline 3 & Finca 18 H.U.M & Paniagua & 51,36 & 15 días después de cosecha \\
\hline 4 & La Ligia & Coyoles & 3,50 & 15 días después de cosecha \\
\hline 5 & La Ligia & $\begin{array}{l}\text { Río Melón Bajo - } \\
\text { Barbudal }\end{array}$ & 10,60 & Siete días después de cosecha \\
\hline 6 & La Ligia & Misteriosa & 17,00 & Embuchamiento \\
\hline 7 & La Ligia & Cola de venado & 18,50 & Floración \\
\hline 8 & La Ligia & $\begin{array}{l}\text { Naranjo Bajo - } \\
\text { Maternidad Bajo }\end{array}$ & 33,30 & Un mes después de cosecha \\
\hline 9 & El Diputado & El Diputado & 37,00 & Primordio \\
\hline 10 & El Diputado & La Plaza & 13,00 & Primordio \\
\hline 11 & El Diputado & Los Zúñiga 1 & 30,00 & Maduración \\
\hline 12 & El Diputado & Los Zúñiga 2 & 40,00 & Maduración \\
\hline 13 & $\begin{array}{l}\text { Hacienda Pelón } \\
\text { de la Bajura }\end{array}$ & Jobo Cutacha & 142,00 & Un mes después de cosecha \\
\hline 14 & $\begin{array}{l}\text { Hacienda Pelón } \\
\text { de la Bajura }\end{array}$ & Río Seco 3 & 70,00 & Arroz voluntario \\
\hline 15 & $\begin{array}{l}\text { Hacienda Pelón } \\
\text { de la Bajura }\end{array}$ & Bambú 1 y 2 & 140,00 & Arroz voluntario \\
\hline 16 & $\begin{array}{l}\text { Hacienda Pelón } \\
\text { de la Bajura }\end{array}$ & Parcelas & 40,00 & Macollamiento \\
\hline
\end{tabular}

En cada campo se identificaron y seleccionaron sitios con vegetación en: bordes, entrada maquinaria, interior y canal de drenaje. Para cada sitio, se estableció un punto de muestreo circular de ocho metros de radio, es decir de aproximadamente $201 \mathrm{~m}^{2}$; y se recolectaron en cada punto tres plantas por especie silvestre presente. Mediante uso de bisturí, se tomaron de cada planta tres muestras (cuatro centímetros cada una) de: vainas de hojas, inflorescencias y frutos, para la búsqueda de $S$. spinki.

Cada una de las muestras se observaron a través del estereoscopio (Olympus Tokio Japan 219719) a 20X, en búsqueda de S. spinki. Los individuos fueron capturados mediante utilización de pincel de punta fina (número 00). Los huevos, estados inmaduros y adultos del ácaro fueron contados y depositados en frascos con alcohol al 75 \% como medio de preservación. 
Montajes e identificación taxonómica de $S$. spinki

Los individuos capturados y colocados en medio de preservación (Alcohol $75 \%$ ), se llevaron al Laboratorio de Entomología de la Universidad Nacional - Sede Central, para los respectivos montajes en medio Hoyer. Debido a que los ácaros fueron conservados en alcohol al $75 \%$, se lavaron con agua destilada al momento de hacer los respectivos montajes, para evitar encogimientos posteriores. En una gota de la solución del medio del montaje puesta en el centro del portaobjetos, se colocaron hasta cinco ácaros y se les hizo descender con ayuda de la punta de un pincel fino al fondo de la gota; allí quedaron en la forma y orden deseados. Inmediatamente se taparon con un cubreobjetos circular de $12 \mathrm{~mm}$ de diámetro. Luego de montar una serie de láminas correspondientes a la misma muestra se procedió a rotular las laminillas. Se colocaron dos etiquetas; en la de la izquierda se escribieron los datos de recolección con tinta indeleble (fecha, localidad, hospedero, colector), y en la de la derecha, el número de colección y la identificación. Finalmente las láminas fueron secadas en estufa a $45^{\circ} \mathrm{C}$ durante cuatro días, y, posteriormente selladas y almacenadas en posición horizontal dentro de cajas de madera para colecciones de ácaros.

La identificación de la especie, se realizó mediante la clave de Smiley et al. (1993). Esta clave ilustrada incluye especies de ácaros del género Steneotarsonemus (Familia Tarsonemidae) que son plagas en el hemisferio occidental, tales como: $S$. brasiliensis, $S$. hyaleos Beer, S. furcatus De León, S. friedmani Smiley, S. bancrofti (Michael), S. spinki Smiley, S. kruseae Ochoa, S. konoi Smiley, S. phyllophorus (Ewing), S. paspali De León, y $S$. spirifex Marchal.

\section{Caracterización poblacional}

Las características poblacionales de S. spinki en plantas hospedantes fueron evaluadas mediante las siguientes variables: número de individuos/ estadio en hojas, flores y frutos. Se realizó el conteo total de individuos por estadios encontrados en las muestras de plantas tomadas, para cada especie silvestre, y se evaluaron las siguientes variables: número total de huevos, número de individuos en estados inmaduros, hembras adultas y machos adultos encontrados.

Para el análisis de la información recopilada se utilizó una técnica de estadística descriptiva que permitió determinar diferencias entre: preferencia del ácaro por plantas hospedantes encontradas y número de especies hospedantes en las regiones arroceras muestreadas. Con la información obtenida de la presencia de S. spinkiy número de individuos por estadio encontrados en vainas de hojas, inflorescencias y frutos, se determinó el hábitat preferencial del ácaro en cada especie hospedante y la proporción poblacional entre cada fase del ciclo de desarrollo y la población total encontrada.

Finalmente, de acuerdo con la información obtenida, fueron seleccionadas las dos especies de plantas arvenses con mayor potencial hospedante para S. spinki. Estas dos especies fueron evaluadas posteriormente, para determinar la duración del ciclo de vida del ácaro y así poder concluir acerca de la existencia de plantas hospedantes en las zonas arroceras de Costa Rica, que permiten que el ácaro sobreviva en épocas donde no están establecidos los campos de arroz.

\section{RESULTADOS Y DISCUSIÓN}

\section{Muestreo}

Se realizó la búsqueda de $S$. spinki en 60 especies de plantas asociadas a 16 campos de arroz en Costa Rica, de las que cuatro especies de arvenses (plantas que crecen en forma silvestre en campos cultivados o ambientes antropogénicos).

Su presencia puede tener efectos negativos o no sobre el cultivo. Malezas de importancia en el cultivo de arroz, pertenecientes a la 
familia Poaceae, presentaron presencia de S. spinki. Las familias botánicas con mayor número de especies muestreadas fueron Poaceae, Cyperaceae y Asteraceae, con 22, 11 y 5 especies, respectivamente (Cuadro 3).

Cuadro 3. Relación de plantas muestreadas y plantas con presencia de S. spinki en campos de arroz. 2007.

\begin{tabular}{|c|c|c|c|}
\hline Nombre científico & Familia & Nombre común & S. spinki \\
\hline Alternanthera polygonoides (L.) R. Br. & Amaranthaceae & Botoncillo & - \\
\hline Amaranhus spinosus L. & Amaranthaceae & Bledo espinoso & - \\
\hline Anthephora hermaphrodita (L.) Kuntze & Poaceae & Falso abrojo & - \\
\hline Arachis pintoi Krapov. \& W.C. Gregory & Fabaceae & Maní forrajero & - \\
\hline Canna indica L. & Cannaceae & Achira & - \\
\hline Caperonia palustris (L.) A. St.-Hil. & Euphorbiaceae & Caperonia & - \\
\hline Cassia tora L. & Fabaceae & Casia & - \\
\hline Chloris radiata (L.) Sw. & Poaceae & Cola de zorro & - \\
\hline Cleome viscosa $\mathrm{L}$. & Capparaceae & Cleome & - \\
\hline Clidemia hirta (L.) D. Don. & Melastomataceae & Peludo & - \\
\hline Cocos nucifera L. & Arecaceae & Coco & - \\
\hline Commelina diffusa Burm. $\mathrm{f}$. & Commelinaceae & Hierba de pollo & - \\
\hline Cynodon dactylon (L.) Pers. & Poaceae & Zacate bermuda & - \\
\hline Cynodon nlemfuensis Vanderyst & Poaceae & Estrella & - \\
\hline Cyperus diffusus Vahl & Cyperaceae & Tres filos & - \\
\hline Cyperus ferax Rich. & Cyperaceae & Coyolillo & - \\
\hline Cyperus flavus J. Presl \& C. Presl & Cyperaceae & & - \\
\hline Cyperus iria L. & Cyperaceae & Sontol & - \\
\hline Cyperus luzulae (L.) Rottb. ex Retz. & Cyperaceae & Coyolillo & - \\
\hline Cyperus rotundus $\mathrm{L}$. & Cyperaceae & Coyolillo & - \\
\hline Dactyloctenium aegyptium (L.) Willd. & Poaceae & & - \\
\hline Digitaria sp. & Poaceae & Alambrillo & - \\
\hline Echinochloa colona (L.) Link & Poaceae & Arrocillo & + \\
\hline Eclipta alba (L.) Hassk. & Asteraceae & Botoncillo & - \\
\hline Eleusine indica (L.) Gaertn & Poaceae & Pata de gallina & + \\
\hline Emilia fosbergii Nicolson & Asteraceae & Clavelillo & - \\
\hline Erechtites hieraciifolius (L.) Raf. Ex DC. & Asteraceae & Hierba de cabro & - \\
\hline Fimbristylis dichotoma (L.) Vahl. & Cyperaceae & Pelo de chino & - \\
\hline Fimbristylis miliacea (L.) Vahl. & Cyperaceae & Pelo de chino & - \\
\hline Gynerium sagittatum (Aubl.) P. Beauv. & Poaceae & Caña brava & - \\
\hline Hedychium coronarium J. König & Zingiberaceae & & - \\
\hline Heliotropium indicum L. & Boraginaceae & Alacrancillo & - \\
\hline Hemidiodia ocymifolia (Willd. Ex Roem. \& Schult.) K. Schum & Rubiaceae & Chiquizá & - \\
\hline Heteranthera limosa (Sw.) Willd. & Pontederiaceae & Lirio & - \\
\hline Homolepis aturensis (Kunth) Chase & Poaceae & Zacate amargo & - \\
\hline
\end{tabular}




\begin{tabular}{|c|c|c|c|}
\hline Nombre científico & Familia & Nombre común & S. spinki \\
\hline Ischaemum rugosum Salisb. & Poaceae & Zacate manchado & - \\
\hline Kyllinga brevifolia Rottb. & Cyperaceae & Fosforito & - \\
\hline Leptochloa filiformis (Pers.) P. Beauv. & Poaceae & Plumilla & - \\
\hline Leptochloa uninervia (J. Presl.) Hitchc. \& Chase & Poaceae & Plumilla & - \\
\hline Ludwigia octovalvis (Jacq.) P.H. Raven & Onagraceae & Clavito & - \\
\hline Ludwigia sericea (Cambess.) H. Hara & Onagraceae & Clavito & - \\
\hline Mimosa pigra L. & Fabaceae & Zarza & - \\
\hline Murdannia nudiflora (L.) Brenan & Commelinaceae & Cangrejillo & - \\
\hline Oryza sativa L. & Poaceae & Arroz & + \\
\hline Oryza latifolia Desv. & Poaceae & Arroz pato & + \\
\hline Panicum maximum Jacq. & Poaceae & Pasto guinea & - \\
\hline Paspalum fasciculatum Willd. Ex Flüggé & Poaceae & Gamalote & - \\
\hline Paspalum virgatum $\mathrm{L}$. & Poaceae & Zacate de burro & - \\
\hline Peperomia pellucida (L.) Kunth & Piperaceae & Lombricilla & - \\
\hline Phyllanthus niruri L. & Euphorbiaceae & Tamarindillo & - \\
\hline Physalis angulata $\mathrm{L}$. & Solanaceae & Farolito chino & - \\
\hline Pseudelephantopus spicatus (B. Juss. Ex Aubl.) C.F. Baker & Asteraceae & Oreja de burro & - \\
\hline Rottboellia cochinchinensis (Lour.) Clayton & Poaceae & Zacate indio & + \\
\hline Saccharum officinarum L. & Poaceae & Caña de azúcar & - \\
\hline Scleria melaleuca Rchb. ex Schltdl. \& Cham. & Cyperaceae & Navajuela & - \\
\hline Scleria pterota C. Presl. & Cyperaceae & Quiebra muela & - \\
\hline Sida acuta Burm. f. & Malvaceae & Escobilla & - \\
\hline Sorghum bicolor (L.) Moench & Poaceae & Sorgo & - \\
\hline Tridax procumbens $\mathrm{L}$. & Asteraceae & Hierba del toro & - \\
\hline Urochloa mollis (Sw.) Morrone \& Zuloaga & Poaceae & & - \\
\hline
\end{tabular}

De las especies arvenses encontradas con presencia de $S$. spinki, E. colona tuvo la mayor frecuencia de muestreo con un total de 90 plantas, de las que se evaluaron 270 muestras de vainas de hojas e igual número de inflorescencias. En las plantas positivas, el número máximo de individuos encontrados fue de 17 y el mínimo de uno.

La especie $O$. latifolia, fue la arvense que presentó el mayor número de individuos de S. spinki. En total fueron muestreadas 60 plantas de la especie, y evaluadas 180 muestras de vainas de hojas e igual número de inflorescencias. El número de individuos por planta encontrado osciló entre 1 y 118.
De las arvenses E. indica y $R$. cochinchinensis, fueron muestreadas 54 y 39 plantas, respectivamente. Las plantas con presencia de $S$. spinki presentaron entre uno y tres individuos por planta.

El hábitat preferencial de $S$. spinki en las plantas hospedantes encontradas fueron las vainas de hojas, y solamente en dos ocasiones fueron halladas hembras en inflorescencias de E. colona. La mayor presencia del ácaro se observó en áreas de bordes de los campos evaluados, seguido de los sitios por donde ingresa la maquinaria a realizar labores agrícolas. 


\section{Caracterización poblacional}

Los muestreos sobre plantas de arroz comercial y residuos de cosecha de $O$. sativa, indicaron densidades de $S$. spinki para las regiones Brunca, Pacífico Central, Huetar Atlántico y Chorotega de 37,80; 23,10; 34,20; 35,70 ácaros/ planta, respectivamente (Figura 2). Es necesario aclarar que el valor promedio por planta, se toma teniendo en cuenta solamente las poblaciones encontradas en $12 \mathrm{~cm}$ de longitud provenientes de tres vainas de hojas evaluadas. En general, la densidad del ácaro en plantaciones de arroz comercial establecidas es de 28,90 ácaros/ planta y para residuos de cosecha (socas) de 34,80 ácaros/ planta.

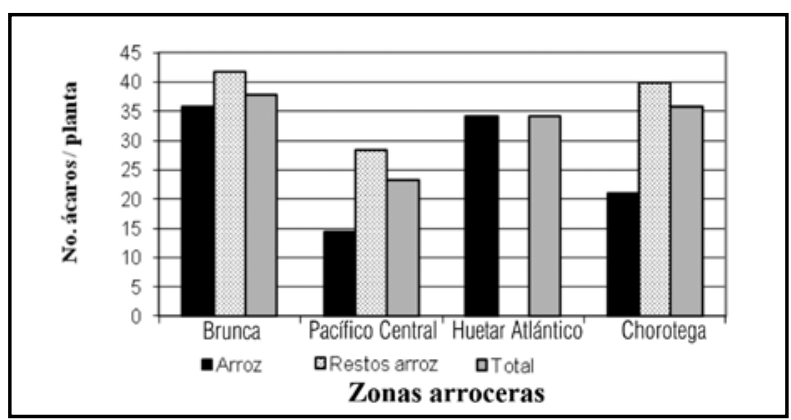

Figura 2. Densidad poblacional de $S$. spinki en Oryza sativa, en cuatro zonas arroceras de Costa Rica. 2007.

En el Cuadro 4 se muestra el número total de individuos encontrados, número de huevos, estados inmaduros (larvas activas + larvas inactivas), adultos hembras y adultos machos, hallados en plantas de arroz comercial y en especies arvenses asociadas a campos de arroz. Solamente en $O$. sativa y $O$. latifolia se encontraron todos los estados del ciclo de vida de $S$. spinki, y con menor densidad poblacional en O. latifolia. En E. colona hubo presencia de hembras adultas del ácaro (uno a cuatro individuos/ planta), y ocasionalmente poco número de huevos (uno a tres huevos/ planta). Solamente fue posible la observación de una larva móvil sobre vainas de hojas de la especie. En E. indica y $R$. cochinchinensis se encontraron hembras de $S$. spinki, en baja densidad poblacional (uno a tres individuos/ planta).
Cuadro 4. Presencia de S. spinkien arvenses asociadas al cultivo del arroz en cuatro zonas arroceras de Costa Rica. 2007.

\begin{tabular}{l|c|c|c|c|c}
\hline $\begin{array}{l}\text { Especie } \\
\text { hospedante }\end{array}$ & Huevos & $\begin{array}{c}\text { Estados } \\
\text { inmaduros }\end{array}$ & Machos & Hembras & TOTAL \\
\hline $\begin{array}{l}\text { Oryza } \\
\text { sativa }\end{array}$ & 1.733 & 1.608 & 435 & 584 & 4.360 \\
\hline $\begin{array}{l}\text { Oryza } \\
\text { latifolia }\end{array}$ & 126 & 377 & 57 & 234 & 794 \\
\hline $\begin{array}{l}\text { Echinochloa } \\
\text { colona }\end{array}$ & 9 & 1 & 0 & 23 & 33 \\
\hline $\begin{array}{l}\text { Eleusine } \\
\text { indica }\end{array}$ & 0 & 0 & 0 & 8 & 8 \\
\hline $\begin{array}{l}\text { Rottboellia } \\
\text { cochinchinensis }\end{array}$ & 0 & 0 & 0 & 7 & 7 \\
\hline
\end{tabular}

En este trabajo se encontró que $S$. spinki es abundante en $O$. sativa y $O$. latifolia, y se presenta ocasionalmente y en bajas densidades poblacionales en las otras tres especies arvenses asociadas a los campos de arroz en Costa Rica. La densidad de S. spinki, referida como el número de ácaros por plantas muestreadas, aparecen en el Cuadro 5.

Cuadro 5. Densidad de S. spinki por especie vegetal encontrada en cuatro zonas arroceras de Costa Rica. 2007.

\begin{tabular}{l|c|c}
\hline Especie & $\mathbf{n}$ & $\begin{array}{c}\text { Número } \\
\text { individuos/ } \mathbf{~}\end{array}$ \\
\hline Oryza sativa & 138 & 31,60 \\
\hline Oryza latifolia & 60 & 13,20 \\
\hline Echinochloa colona & 90 & 0,40 \\
\hline $\begin{array}{l}\text { Rottboellia } \\
\text { cochinchinensis }\end{array}$ & 39 & 0,20 \\
\hline Eleusine indica & 54 & 0,10 \\
\hline
\end{tabular}

Se destacó la alta densidad del ácaro $(13,20)$ en todos sus estados de desarrollo sobre la arvense 0 . latifolia, así como la presencia de tres estados de desarrollo (huevo, larva móvil y adultos hembras) en E. colona. La baja densidad de $S$. spinki en $E$. indica y $R$. cochinchinensis y la presencia de solamente 
hembras, sugirió que estas dos especies arvenses son hospedantes ocasionales 0 accidentales, resultado de los mecanismos (viento, maquinaria, y lluvia) de transporte del ácaro en los campos de arroz.

En los análisis gráficos de la estructura de población de $S$. spinki en plantas hospedantes (Figura. 3), se observó que en $O$. sativa y $O$. latifolia hubo una tendencia similar en el porcentaje de individuos por planta que corresponden a machos adultos y estados inmaduros, y marcada diferencia en el porcentaje de hembras y huevos encontrados, con respecto a la población total que se encontró en cada especie. En E. colona el mayor porcentaje de la población fueron hembras adultas; se observó bajo número de huevos y estados inmaduros, y no hubo presencia de machos adultos.

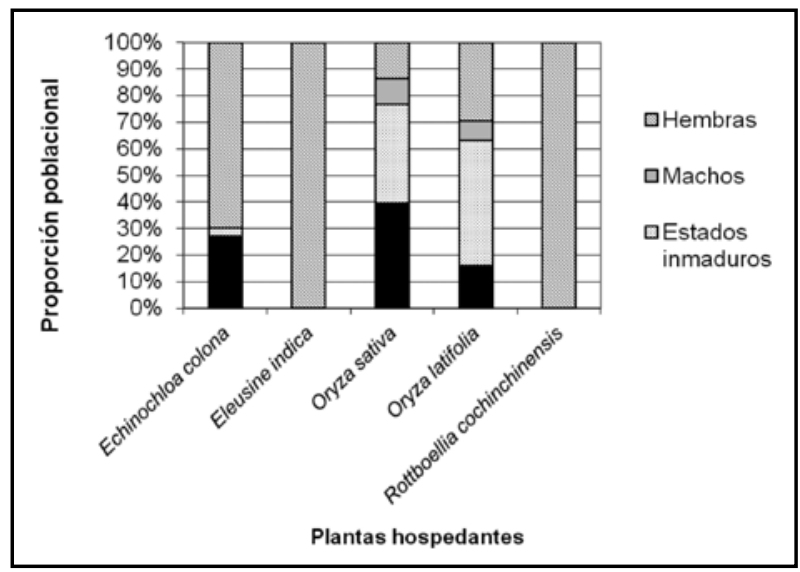

Figura 3. Proporción poblacional de S. spinki en plantas hospedantes arvenses presentes en agroecosistemas de arroz en Costa Rica. 2007.

\section{DISCUSIÓN Y CONCLUSIONES}

El ácaro presente en las vainas de arroz y arvenses asociadas a los agroecosistemas muestreados de cuatro zonas de Costa Rica fue identificado como $S$. spinki. En el interior de las vainas de arroz se observaron zonas necrosadas con altos niveles de la población del ácaro (huevos, estados inmaduros, adultos). De acuerdo con la metodología de muestreo empleada, se registraron las mayores poblaciones del ácaro en $O$. sativa, con un promedio general de 2,70 ácaros/ $\mathrm{cm}^{2}$. Sin embargo, este valor es bajo si se compara con algunos reportes que indicaron poblaciones promedio de 300 ácaros/ $\mathrm{cm}^{2}$ en las hojas banderas y de 85 ácaros/ $\mathrm{cm}^{2}$ en el resto de las vainas (Socorro y Almaguel 1997). La baja densidad del ácaro encontrada en plantas de arroz, se debió probablemente a que todos los campos muestreados habían sido sometidos días antes del muestreo, a combate químico del ácaro.

Se denomina arroz rojo a algunas poblaciones de $O$. sativa que se comportan como malezas en los agroecosistemas cultivados con arroz comercial. Es la maleza más dañina en los campos arroceros de todo el mundo, debido a su agresividad y difícil manejo (Diarra et al. 1985). El arroz rojo Oryza sativa L., es muy susceptible al ácaro, por lo que es común observar en éste altas infestaciones del ácaro en plantas que crecen en los camellones y bordes de los canales en campos de Costa Rica (Álvarez 2004). En el presente trabajo no se realizaron muestreos al arroz rojo, pues el objetivo de la investigación eran aquellas especies diferentes a $O$. sativa que pudieran hospedar de manera casual o alterna a $S$. spinki.

Con respecto a la presencia del ácaro en especies distintas al arroz, Álvarez (2004), concluyó que $S$. spinki sólo se desarrolla en el género Oryza (arroces contaminantes y arroz rojo). Igualmente, Socorro y Almaguel (1997), informaron en su momento, que en los muestreos realizados en Cuba sobre plantas asociadas y botánicamente similares al arroz, no se ha encontrado otros hospedantes. Los mismos investigadores concluyeron que según los conocimientos adquiridos hasta esa fecha, no era probable que en las condiciones de cultivo de dicho país (arroz todo el año) aparecieran arvenses infectadas por esta plaga. Sin embargo, Torre et al. (2005), detectaron a S. spinki en plantas de E. colona, Panicum purpureans y Amaranthus spinosus. 
Fue interesante haberlo encontrado dentro de inflorescencias de $A$. spinosus, pues se tenían informes de que estos ácaros sólo habitaban dentro de vainas de las hojas de gramíneas. Los individuos de esa muestra estaban perfectamente formados y en buena población de ambos sexos. Por lo anterior, es necesario que en los campos con incidencia de S. spinki, se realicen periódicamente muestreos sobre plantas asociadas a los agroecosistemas, pues posiblemente la necesidad extrema de fuentes alimenticias hizo que el ácaro emigre a otras especies y se adapte temporalmente a nuevas condiciones alimenticias y de hábitat.

Las arvenses causan daños directos en el cultivo de arroz por la competencia de luz, agua y nutrimentos, lo que provoca disminución en la cosecha; e indirectos porque pueden ser hospederas de insectosplaga, ácaros-plaga y enfermedades; y algunas producen compuestos alelopáticos que afectan el crecimiento normal del cultivo. En los agroecosistemas arroceros evaluados, las comunidades de arvenses presentaron mayor número de especies y familias en los bordes y canales de riego; por lo que estos sitios se convirtieron en hábitat ocasional para la sobrevivencia de $S$. spinki en momentos en los que $O$. sativa no estaba presente.

Se determinaron en campo como plantas hospedantes las arvenses: $O$. latifolia, $E$. colona, E. indica y $R$. cochinchinensis. De acuerdo con González et al. (1985) y Lallana (1989), en Latinoamérica, E. colona y E. indica se consideran especies agresivas y frecuentes, que ocasionan pérdidas significativas en campos de arroz. Suárez et al. (2004) incluye dentro de este importante grupo, la arvense $R$. cochinchinensis, la cual es una Poaceae anual, de crecimiento rápido y altamente competitiva con el cultivo de arroz. Estas especies arvenses con presencia del ácaro $S$. spinki, se presentaron con mayor frecuencia en los bordes, convirtiéndose en fuentes de infestación del ácaro hacia el campo de cultivo.

Informes de Santos et al. (2004), indican que durante el año 2004, fueron recolectados huevos, larvas y ninfas de S. spinki sobre la planta invasora O. latifolia, común en los cultivos de arroz en Costa Rica y Panamá; y concluyó que $S$. spinki completó su ciclo de vida en ese hospedero. Sin embargo, el informe no detalló las características poblacionales encontradas de S. spinki sobre la especie hospedante.

Los resultados que se obtuvieron en la presente investigación, con respecto a las características hospedantes de la arvense O. latifolia, confirmaron que las condiciones de hábitat y alimentación permitieron la culminación del ciclo de vida de S. spinki. El número de hembras que se encontró en $O$. latifolia fue relativamente mayor al encontrado en $O$. sativa, debido quizás a que gran porcentaje de los campos evaluados se encontraban en etapas finales de cosecha. Bajo estas condiciones las hembras del ácaro migraron a hospedantes ocasionales y alternos por modificaciones en las condiciones de hábitat y alimentación en las plantas de arroz. Cuando se analizó en $O$. latifolia el porcentaje de la población total que se encontró corresponde a machos y estados inmaduros, existió similitud con la encontrada en O. sativa. El porcentaje de huevos encontrado de manera general en los muestreos, indicaron menor número de huevos en $O$. latifolia. Con respecto al número de individuos total encontrado en las especies hospedantes del género Oryza, se pudo concluir que $O$. latifolia, hospedó un 41,90 \% de la población de $S$. spinki que normalmente se había encontrado en $O$. sativa.

Se determinó la presencia del ácaro en la especie $E$. colona, hallándose hembras, huevos y para un caso una larva móvil, lo que indicó que dentro de la gran diversidad de arvenses en los agroecosistemas arroceros tropicales, quizás esta especie se aproxima a los requerimientos nutricionales y de hábitat de S. spinki. Bajo las condiciones de Cuba, $S$. spinki también se detectó en plantas de $E$. colona con escaso número de ejemplares, por lo que la presencia del ácaro en esta especie fue descrita como algo casual o accidental (Torre et al. 2005). Fue necesario realizar estudios de sobrevivencia del ácaro sobre la 
arvense, para determinar si se trata de una planta hospedante alterna u ocasional que permite la reproducción o sobrevivencia del ácaro.

En la República Popular de China, Socorro y Almaguel (1997) informaron que el ácaro presenta como hospedantes silvestres a $E$. indica y otras cinco especies de la familia Poaceae. En este país a partir de noviembre no hay plantaciones de arroz que puedan garantizar la sobrevivencia del ácaro durante el invierno, por lo que bajo esas condiciones la población del ácaro (especialmente las hembras adultas), migran a estos hospedantes silvestres para sobrevivir y no se desarrollan hasta el inicio de la primavera cuando se restablecen las condiciones favorables para su multiplicación. La arvense $R$. cochinchinensis no había sido informada como hospedante de S. spinki en otras investigaciones.

En las arvenses E. indica y R. cochinchinensis, los resultados indicaron que la presencia del ácaro no es frecuente, debido posiblemente a la insuficiente condición nutricional, por lo que no se hospeda por largo tiempo. No se observó evidencia posible de que pueda producir otra generación, pues los individuos son adultos hembras que se movían rápido. Similares resultados fueron encontrados por Kang-Chen y Chyi-Chen (1977) en Taiwán, quienes realizaron un muestreo de plantas gramíneas, capa de suelo superficial, tallo y plantas de retoño, después de la segunda cosecha. Se encontró en la superficie del suelo que el ácaro $S$. spinki son casi todos adultos hembras. El cuerpo es más pequeño y flaco y se mueve rápido, en busca de plantas para hospedarse, por lo que el informe incluye datos que corresponden solamente a hembras inmigrantes.

Es necesario tener en cuenta que la presencia de hembras $S$. spinki en las especies $E$. indica y $R$. cochinchinensis, no fue suficiente para determinar que sea o no una planta hospedante de importancia en el desarrollo o biología del ácaro. La diseminación del ácaro en el campo, se da de una planta a otra por medio de fuertes vientos, por el agua y por otros insectos; por lo que la presencia del ácaro en estas arvenses, pudo ser resultado del azar en la diseminación provocada por estos medios. Por otro lado, se debe tener en cuenta el hecho de que $E$. indica fue reportada anteriormente con presencia de $S$. spinki (Socorro y Almaguel 1997), bajo las mismas características poblacionales (solo hembras adultas), lo que hizo pensar que posiblemente esta especie fue utilizada como un hospedante que permitió la sobrevivencia por cortos lapsos de tiempo, mientras se establecieron nuevas plantas de arroz en los campos.

Muestreos realizados en zonas arroceras de la India por Rao y Prakash (2002), indicaron la presencia de el ácaro $S$. spinki en Schoenoplectus articulatus (Linn.) Palla. En los muestreos efectuados en el presente trabajo, fueron muestreadas diez especies pertenecientes a la familia Cyperaceae, las cuales presentaron alta frecuencia en todos los agroecosistemas muestreados; sin embargo, no se obtuvo evidencia de colonización de $S$. spinki en ninguna de ellas.

Dentro de la diversidad de arvenses que se encontraron en los agroecosistemas arroceros de Cosa Rica, sólo cuatro especies interaccionaron con S. spinki. Por lo anterior, se puede concluir que la práctica cultural de eliminación total de arvenses en los campos quedó en duda, pues, los planes de manejo de arvenses, debieron tener en cuenta que si determinada especie no se comporta como hospedante alterna, debe analizarse la función de dicha especie como fuente de alimentación y hábitat de los enemigos naturales del ácaro.

Es necesario fortalecer las técnicas de manejo de restos de cosecha en los campos arroceros evaluados. El estudio permitió determinar que las partes vivas de plantas cosechadas, se convirtieron en los principales focos de sobrevivencia e infestación de $S$. spinki, en los momentos del año en los que no hay plantaciones de arroz establecidas. 
La proporción de hembras encontradas, con respecto a las poblaciones totales de $S$. spinki en las especies $E$ colona, E. indica y $R$. cochinchinensis, indicaron 70, 100 y $100 \%$, respectivamente. Los resultados coincidieron con lo informado por Almaguel et al. (2003), y se explicaron por la función biológica de las hembras de garantizar la reproducción sexual del ácaro, por lo que emigran a plantas hospedantes ocasionales.

\section{RECOMEDACIONES}

Continuar con el diseño y desarrollo de investigaciones que tengan como objetivo fortalecer las estrategias de combate de $O$. latifolia y los distintos fenotipos de $O$. sativa agrupados como arroz rojo en campos arroceros de Costa Rica. La cercanía genética, y en especial la morfología y fisiología de estas arvenses, facilitan el desarrollo de las poblaciones de $S$. spinki, e igualmente dificultan su combate en las plantaciones de arroz.

Realizar ensayos que permitan evaluar la sobrevivencia, desarrollo y reproducción de $S$. spinki en las plantas hospedantes $O$. latifolia y E. colona. Lo anterior, con el objetivo de determinar el potencial que ofrecen estas especies para aumentar las poblaciones del ácaro plaga.

\section{AGRADECIMIENTOS}

Los autores agradecen a las instituciones costarricenses: Instituto Nacional de Innovación y Transferencia de Tecnología Agropecuaria (INTA), Corporación Arrocera Nacional (CONARROZ), y a la Escuela de Ciencias Agrarias de la Universidad Nacional, Costa Rica, por su financiación y colaboración en las actividades de campo y laboratorio desarrolladas. Al personal administrativo y de campo de Finca 18 Haciendas Unidas Mecanizadas, Hacienda La Ligia, Finca El Diputado y Hacienda El Pelón de la Bajura, por su enorme contribución a las labores de muestreo en los campos de arroz.

\section{LITERATURA CITADA}

Almaguel, L.; Santos, A.; Torre, PE de la; Botta, E.; Hernández, J.; Cáceres, I.; Ginarte, A. 2003. Dinámica de población e indicadores ecológicos del ácaro Steneotarsonemus spinki Smiley 1968 (Acari: Tarsonemidae) en arroz de riego en Cuba. Fitosanidad 7(1): 23-30.

Almaguel R., L. 2002. Curso introductorio a la acarología aplicada: morfología, taxonomía y diagnóstico fitosanitario de ácaros de importancia agrícola. Instituto de Investigaciones de Sanidad Vegetal (INISAV). ISBN: 959-7111-24-1. Ciudad de la Habana, Cuba. p. 84.

Álvarez B., F. 2004. Apreciaciones sobre el ácaro blanco del arroz (Steneotarsonemus spinki Smiley). Informe Servicio Fitosanitario del Estado, MAG (Costa Rica), 24 de junio de 2004. p 3.

Barquero S., M. 2004. Limón y Guanacaste: Severo ataque de ácaro del arroz. La Nación, San José, Costa Rica, agosto 3.

Diarra, A.; Smith, R.; Talbert, R. 1985. Interference of red rice (Oryza sativa) with rice (Oryza sativa). Weed Sci. 33: 644-649.

González, J.; Arregoces, O.; Escobar, E. 1985. Principales malezas en el cultivo de arroz en América Latina. In Arroz: investigación y producción. Capítulo VII. CIAT. Cali, Colombia. p. 419444.

Hilje, L. 1995. Siete preguntas de actualidad sobre el manejo integrado de plagas en América Central. Agronomía Mesoamericana 6: 169-178. 
Holdridge, L.R. 1993. Mapa ecológico de Costa Rica según el sistema de clasificación de zonas de vida del mundo. Centro Científico Tropical (CCT)/ Ministerio de Agricultura y Ganadería (MAG). p 72.

Kang-Chen, L; Chyi-Chen, H. 1977. Estudio sobre tarsonémido Steneotarsonemus spinki (Acarina : Tarsonemidae). National Science Council Monthly 4(4): $21 \mathrm{p}$.

Lallana, V. H. 1989. Malezas el arroz en Sudamérica. Rev. Facultad de Agronomía, UBA. 10(1-2): 87-94.

Rao, J.; Prakash, 2002. A. paddy field leed, Schoenoplectus articulatus (Linn.) Palla (Cyperaceae): a new host of tarsonemidae mite, Steneotarsonemus spinki Smiley and panicle thrips, Haplothrips ganglbaureri Schmutz. Journal of Applied Zoological Researches, 13(2): 174-175.

Reyes H., L. A. 2005. Ácaro del vaneamiento del arroz Steneotarsonemus spinki Smiley (Prostigmata: Tarsonemidae). CIAT. Disponible en: http://www.flar. org/pdf/foro-agosto-pdf05/acaro.pdf. Consultado el 10 de febrero de 2007.

Santos M., R.; Navia, D.; Cabrera, R. I. 2004. Steneotarsonemus spinki Smiley (Acari: Prostigmata: Tarsonemidae) una amenaza para el cultivo de arroz en Brasil. EMBRAPA (Empresa Brasileira de Pesquisa Agropecuaria). Documentos 117. Brasilia, DF. ISSN 01020110. p 54.

Socorro, M.; Almaguel, L. 1997. Informe de la misión técnica sobre el cultivo del arroz a la República Popular China, del 1-15 de noviembre de 1997. p 21.
Smiley, R. L.; Flechtmann , C. H. W.; Ochoa, R. 1993. A new species of Steneotarsonemus (Acari: Tarsonemidae) and an illustrated key to grass-infesting species in the Western Hemisphere. Internat. J. Acarol. 19(1): 87-90. 1993.

Suárez, L.; Anzalone, A.; Moreno, O. 2004. Evaluación del herbicida halosulfuron-metil para el control de malezas en el cultivo de arroz (Oryza sativa L.). Bioagro 16(3): 173-182.

Torre S., PE de la; Botta F., E; Almaguel R., L. 2005. Colectas acarológicas realizadas por la sanidad vegetal en la Provincia de La Habana. Fitosanidad 9(3): 3-11. 
ALCANCES TECNOLÓGICOS, AÑO 6, NÚMERO 1 